\title{
Analysis of Light Propagation in Identical and Non-Identical Multicore Photonic Crystal Fibers
}

\author{
Miami Mohammed 1,2 and Ahmad K. Ahmad ${ }^{3, *}$ \\ ${ }^{1}$ Institute of Applied Physics, University of Muenster, CorrensstrBe 2-4, 48149 Muenster, Germany \\ ${ }^{2}$ Department of Physics, College of Science, University of Al-Mustansiriyah, Baghdad, Iraq \\ ${ }^{3}$ Department of Physics, College of Science, Al-Nahrain University, Baghdad, Iraq
}

\begin{tabular}{ll}
\hline \multicolumn{1}{c}{ Articles Information } & \multicolumn{1}{c}{ Abstract } \\
\hline Received: & Multicore photonic crystal fibers (MPCF) with identical and non-identical cores \\
29.05 .2020 & are analyzed numerically using Comsol Multiphysics software. Investing \\
Accepted: & coupling between cores limiting by parameters, such as the slight change in \\
21.08 .2020 & central core diameter; the separation distance between cores and the \\
Published: & wavelength were investigated. Controlling these parameters allows predicting \\
26.09 .2020 & the mode propagation. Moreover, anisotropy in all cores diameters of multicores \\
& photonic crystal fibers, as a result, the change of all core diameters \\
Keywords: & simultaneously lead to different of coupling behavior, suppresses the coupling \\
Multicore photonic crystal fibers & between the core modes by inducing a small mismatch between the modes such \\
Coupler & that the core modes to be uncoupled through this change. Then this leads to the \\
Multiplexers & light mode propagate independently of their neighbors and gives rise to the \\
De-multiplexers & mode to primarily remain in a certain region of the MPCF array. These \\
& properties suggest that multicore PCFs could be a novel candidate for both \\
& multiplex or de-multiplex.
\end{tabular}

DOI: 10.22401/ANJS.23.3.07

*Corresponding author: ahmad.kamal@sc.nahrainuniv.edu.iq

\section{Introduction}

Photonic crystal fibers (PCFs) are a new class of optical fibers that have emerged in the past years [1,2]. Their design includes a core in the central region surrounded by a periodic array of air gaps centered in the cladding region $[3,4]$. PCF can be divided into different types, depending on the design of its core shape, being either solid or hollow [4,5]. The first, index-guiding PCF guides light by total internal refraction (TIR) between a solid core and a cladding region with multiple air holes [4-6]. The second uses a perfect periodic structure exhibiting a photonic bandgap (BPG) at the operating wavelength to guide light in a low index hollow core-region [4,5]. In this contribution, we will focus on index-guiding PCF, where the index in the core region is slightly higher than the one in the cladding region [7]. The design flexibility of PCF in terms of changing the number, size, shape, and arrangement of holes allows tailoring unique characteristics that cannot be achieved by conventional optical fibers $[7,8]$. PCF can serve in a wide range of applications [7], such as endlessly single-mode operation $[5,9]$, bend-loss edge at short wavelength [5]. Controlled effective-core-area at the single-mode region and anomalous group-velocity dispersion at visible and nearinfrared wavelength $[4,5,10]$, all of which make photonic crystal fibers an ideal candidate for a variety of applications in optical communication systems [1,7].
Recently, developments have revealed that multicore photonic crystal fiber [12], which has attracted interest from researchers for the flexibility of its design, easy manufacturing process, and many research interests are shown towards MPCF couplers [7], the coupling properties of MPCF with adjacent identical cores, useful for coupling [1-15], splitter [16], switching [17-18]. Besides, it possesses the possibility of realizing a multiplexerdemultiplexer (MUX-DEMUX) [5]. When we decided to destroying the symmetry of MCPCF structure is the key point of all method to obtain higher birefringence in MCPCF. Generally, one can happen difference between the cores of PCF and become non-identical; either by using different index profile, or by using different dimensions, causing changing in refractive index distribution, and this leads to a unequal power distribution in the core of MCPCF. The MPCF coupler with nonidentical cores can also be used for coupling $[8,12,19,20]$, switching [17], and to achieving equal group velocities for different cores to reduce intermodal dispersion [20].

In our study, we have discovered the possibility of devising various forms of MCPCF by manipulating of the geometrical design of the structure, such as a s slight change in central core diameter, cores separation and anisotropy in all core diameters of $\mathrm{MCPCF}$, all these changes to predict the mode behavior in several of coupled MCPCF designs, which have the potential to affect the coupling properties between cores or reduce the 


\title{
Al-Nahrain Journal of Science
}

\author{
ANJS, Vol.23 (3), September, 2020, pp. 49 - 60
}

coupling significantly, so that the coupling nonexistent between the cores and the cores become decoupled, thus spreads the light independently mainly in all cores, as individual cores and in isolation, which are limited in single core in a certain region of PCF. We present our theoretical work based on finite element method (FEM) by using the wave module of available COMSOL MULTIPHYSICS software.

\section{Theory and Design Methodology}

We choose to design a seven-core PCF system as MCPCF with different structures, because of the coupling behavior, the seven-core system examined in detail to predict an extensive system. Identical and non-identical seven-core systems, we suppose cores are identical, and each core is to be single-mode with propagation constant is $\beta$, the coupling coefficient between seven-cores is $\kappa$, the evolution of the model field amplitudes $U_{0}$ in the sevencore fibers system, can describe bellow as in equation (1) [21]:

$$
i \frac{d U_{0}}{d z}+\beta U_{0}+\kappa \sum_{n=1}^{6} U_{n}=0
$$

The eigenmodes solution of this system and by assuming the interactions are for nearest neighbor only in this system is $U_{n}=u_{n} \exp (i \beta z)$, which connected with the supermodes. The coupling coefficient $\kappa$ for identical cores and nonidentical cores are explained in detail in $[1,21]$ and [20].

On the other hand, to describe the relationship between the coupling coefficient and the power flow in each core as a function of $\mathrm{z}$ according to coupled-mode theory (CMT), when two waveguides are identical, called them $\mathrm{U}_{1}, \mathrm{U}_{2}$. The coupling of the evanescent field of waveguides can be described as equation (2) below [22]:

$$
\begin{aligned}
& \frac{d U_{1}(z)}{d z}=i \beta_{1} U_{1}(z)+\kappa_{12} U_{2}(z) \\
& \frac{d U_{2}(z)}{d z}=i \beta_{2} U_{2}(z)+\kappa_{21} U_{1}(z)
\end{aligned}
$$

The amplitude of these waveguides is $\mathrm{U}_{1}$ and $\mathrm{U}_{2}$ accompanied by continuous propagation such as $\beta_{1}$ and $\beta_{2}$ for propagation along the $\mathrm{z}$-direction. The coupling coefficient $\kappa_{\mathrm{ij}}$, according to the coupled-mode theory, can be defined as the modal overlap between the waveguides as equation (3) [10]:

$$
\kappa_{12}=\frac{k_{0}^{2}}{2 \beta} \iint_{\text {area }}\left(n_{1}^{2}-n_{2}^{2}\right) F_{1}^{*}(x, y) F_{2}(x, y) d x d y
$$

where the refractive indexes of the waveguide core and cladding are $\mathrm{n}_{1}$ and $\mathrm{n}_{2}$. the modal distribution is $\mathrm{Fi}(x, y)$ and for $(i=1,2) . \beta=1 / 2\left(\beta_{1}+\beta_{2}\right)$ is the average of the modal individual propagation constants. The coupling between two-waveguides or cores can be expressed by the supermode theory that dependence on hybridized patterns, such as two modes either even (symmetric) or odd (antisymmetric) super-mode. The coupling coefficient $\kappa$, which determines the strength of the coupling between the cores is expressed as a perturbation of the individual modes and this provides the value for the mode splitting as $\kappa=1 / 2\left(\beta_{\text {even }}-\beta_{\text {odd }}\right)$, where $\beta_{\text {even }}$ and $\beta_{\text {odd }}$ represent the propagation constants of the even and odd modes. Where the constant propagation $\beta=2 \pi n_{\text {eff }} / \lambda$, and its corresponding effective refractive index $n_{\text {eff }}$ of supermodes [2].
When we have a two-core scenario, it results in two sets of even and odd modes, known as fundamental supermodes, i.e., there are four modes of which are two even and odd modes for both $\mathrm{x}$ - and $\mathrm{y}$-polarized field orientation, such as ( $\left.E_{\text {even }}^{x}\right)$ and $\left(E_{\text {odd }}^{x}\right)$ for $x$-polarization fields. And $\left(E_{\text {even }}^{y}\right)$ and $\left(E_{\text {odd }}^{y}\right)$ for $y$-polarization fields $[3,15]$. They have different propagation constants and effective refractive indices, such as $\beta_{\text {even }}^{x}, \beta_{\text {even }}^{y}, \beta_{\text {odd }}^{x}$, $\beta_{\text {odd }}^{y}$ and $n_{\text {even }}^{x}, n_{\text {even }}^{y}, n_{\text {odd }}^{x}, n_{\text {odd }}^{y}$, respectively. Two modes of propagation in phase are called even modes, and the other two modes of propagation anti-phase called odd modes $[1,3,25]$. Here in our study, we have a seven-core scenario, it results in seven sets of even and odd supermodes, i.e., there are fourteen modes of which are seven even and odd modes for both $\mathrm{x}$ - and $\mathrm{y}$-polarized field orientation

The relation between the coupling length and the coupling coefficient is given in equation (4) as $[3,25,26$, 27]:

$$
\begin{aligned}
L_{c} & =\frac{\pi}{\beta_{\text {even }}-\beta_{\text {odd }}} \\
& =\frac{\lambda}{2\left(n_{\text {even }}-n_{\text {odd }}\right)}
\end{aligned}
$$

The coupling length can define as the minimum longitudinal distance at which all-optical power, when incident initially on one of the fiber cores is periodically transferred from this fiber core to a second core. Or it is defined as the rate of power transfer between cores depends on the core diameter, the core separation, and the wavelength. The coupling efficiency represents the percentage of power transferred between two cores. While can be expressed the power of each core as a function of $\mathrm{z}$ by the Poynting vector using this equation below (5):

$$
P_{\text {core }}(z)=\frac{1}{2 \text { Re } \iint_{\text {corearea }} \vec{E}(x, y, z) \vec{H}^{*}(x, y, z)} \cdot \hat{z} d x d y
$$

The coupling strength and coupling length can quickly determine the via extract of the propagation constants. Then their corresponding refractive indexes numerically using the program Comsol MULTIPHYSICS softwarebased finite element method (FEM)-using the Mode solver model. This provides an easy way to determine the coupling strength and extract the propagation constants, and then their corresponding refractive indexes using numerical simulations using commercial program Comsol MULTIPHYSICS software based finite element method (FEM)-Mode solver model.

FEM solver can analyze the guided modes of the PCF by directly solving Maxwell's equation [7], to obtain an approximate value of the effective refractive index [2], as in equation (6), which represents the vectorial wave equation FEM for the electric field $E$. The effective refractive indices of modes obtained using the module for mode field analysis COMSOL software.

$$
\nabla \times\left(\nabla \mu_{r}^{-1} \times E\right)-k_{0}^{2} \varepsilon_{r} E=0
$$

where $k_{0}$ is represented the free space wave number, $\mu_{r}$ is the permeability of the material, $\varepsilon_{r}$ is the permittivity of the material with $\varepsilon_{r}=n-i k$ and $n$ being the real part of 


\title{
Al-Nahrain Journal of Science
}

\author{
ANJS, Vol.23 (3), September, 2020, pp. 49 - 60
}

the refractive index, $\lambda=j \beta-\sigma z$. By assuming the multicore PCF is a non-magnetic $\mu_{r}=1$ and nonconducting $\sigma=0$. FEM method is used to solve the light propagation in PCFs because of their ability to simulate complex geometric structures, and because it allows the cross- section of the waveguide to divide into a finite triangular mesh which can be of various sizes and shapes [7]. The refractive indexes and modal field distributions evaluated, then, the distributions the power flow as a function of $\mathrm{z}$ in each core of seven cores is determined. After numerically analyzing the mode solver, the refractive indexes, and modal field distributions evaluated, then, making it directly possible to calculate the coupling length $\mathrm{L}_{\mathrm{c}}$, the distributions of the power flow as a function of $\mathrm{z}$ in each core of seven cores are determined.

The modal field distribution of the super-modes is similar to the single-core fiber which has degenerated $\mathrm{HE}_{11}^{\mathrm{X}}$ and $\mathrm{HE}_{11}^{\mathrm{y}}$ modes propagating with the same phase velocity $[1,26]$. Modal field distribution of super-modes in most multicore fiber can provide a deep insight into the properties of their coupling when the cores are identical (weak guiding), the modes treated as a linear polarized (LP), including the 1st fundamental mode LP01 that is degenerate to HE11, and has the largest effective index; LP11 represents the 2 nd and 3rd order modes that are degenerate to TE01, TM01, and HE21 modes. LP02 represents the $4^{\text {th }}$ and $5^{\text {th }}$ order modes that are degenerate to EH11 and HE31 modes. We consider LP11 and LP02 as higher-order modes $[11,25,26]$.

We designed a PCF with identical seven cores, i.e., all the cores have the same diameters of $3.5 \mu \mathrm{m}$. The structure parameters being pitch $\Lambda=5.6 \mu \mathrm{m}$, the hole diameter $4.48 \mu \mathrm{m}$, and the air-filling fraction $\frac{d}{\Lambda}=0.8$ at a wavelength $1.064 \mu \mathrm{m}$. The refractive index of the core $n_{c o}=1.445$ is slightly higher than the refractive index of the cladding $n_{c l}=1.44$. The numerical results show the coupling between the identical seven-core, as depicted in Figure 1.
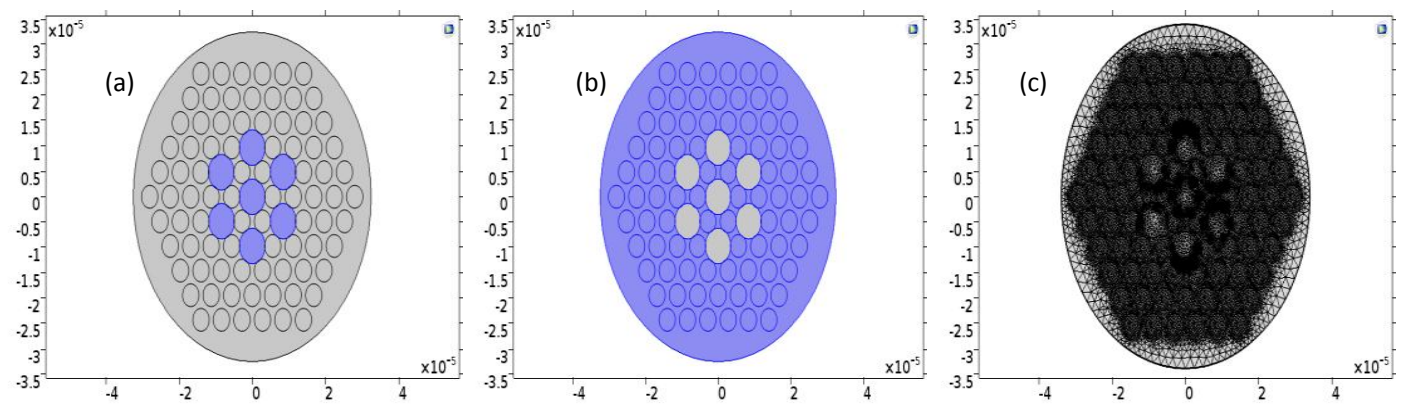

Figure 1. Cross-section of identical seven-core PCF coupler, the geometry of PCF design characterized by the structure parameters, the core diameter $=5 \mu \mathrm{m}$, the hole pitch $\Lambda=5.6 \mu \mathrm{m}$, hole diameter $d=4.48 \mu \mathrm{m}$, the air-filling fraction $d / \Lambda=0.8$, and cores separation $D=2 \Lambda \mu \mathrm{m}$, at the wavelength $\lambda=1.064 \mu \mathrm{m}$. (a) core material, (b) cladding material, (c) Mesh triangular.

\section{Simulation and Discussion}

The numerical results show that by using the effective index for even, and odd modes of coupled seven-core can directly calculated. Figure $2(\mathrm{a} \& \mathrm{~b})$ shows the effective index and electric field along with the $\mathrm{x}$-polarized component $\left(E_{x}\right)$ for both even and odd modes of the sevencore PCF as a first study when all seven cores are identical. The numerical results also show that the modal field distribution can be interpreted as optical power flow in each core of a seven-cores core when the initial input is at the central core, as shown in Figure 2 (c) shows the optical power in each core of a seven-cores when the initial input is at the central core. We notice in this figure three cases that we describe as follows, the first case, the power is limited to the central core, where all seven cores have the same power where the intensity is equal among all cores and leads to excitation of all modes within the central core area as the field distribution of $\mathrm{LP}_{01}$. The second case, the summation all excited modes to the central cores, where all the outer cores have propagation constants that are nearly equal to the propagation constant of the central core, such that the mode field is more confined to the center core, and the coupling of the center core to the neighboring outer cores is weak as the field distribution of $\mathrm{LP}_{02}$. The third case, when the higher order modes are exicted, all the power is centred in the six outer cores as the field distribution of $\mathrm{LP}_{11}$, and the strong coupling between the outers cores (higher-order modes) than the central core. Because the propagation constants of theses cores are equal with each other, we find it shows the strong coupling between the two, three, four and six outers cores.

The second and third cases for non-identical seven-core PCF coupler when the central core have a diameter as $3.27 \mu \mathrm{m}$ and $3.79 \mu \mathrm{m}$, i.e. are slightly smaller or larger than the diameters of the six outer cores with $3.5 \mu \mathrm{m}$. The geometry of design non-identical seven-core PCF with hexagonal lattice characterized by the structure parameters, such as the core diameter $=5 \mu \mathrm{m}$, the hole pitch $\Lambda=5.6 \mu \mathrm{m}$, hole diameter $d=4.48 \mu \mathrm{m}$, the airfilling fraction $d \Lambda=0.8$, and cores separation $D=2 \Lambda \mu \mathrm{m}$, at the wavelength $\lambda=1.064 \mu \mathrm{m}$. Two cases of nonidentical seven core systems are analysed, one when the central core have a diameter of $3.27 \mu \mathrm{m}$ or $3.79 \mu \mathrm{m}$, i.e., 


\title{
Al-Nahrain Journal of Science
}

\author{
ANJS, Vol.23 (3), September, 2020, pp. 49 - 60
}

are slightly smaller or larger than the diameters of the six outer cores as $3.5 \mu \mathrm{m}$. The numerical results show the effective indices for even and odd modes of non-identical coupled seven-core, as shown in Figures 3 and 4(a \& b). The optical power in each core of a non-identical sevencore PCF when the input is initially to the central core, and representation in seven unique power distributions of the fourteen fundamental modes of the seven-core PCF at outface of the fiber as in Figure 3 (c), i.e. when the value of the central core is $3.27 \mu \mathrm{m}$ there was a strong coupling between the central core to three core of the six outer cores that has diameters close to the diameter of the central core and equal propagation constants as the field distribution of $\mathrm{LP}_{12}$, while the residual cores remain without any coupling and participate very little in the power exchange with the others. As well as, a strong overlap between the modes of the central core and outers cores as the field distribution of $\mathrm{LP}_{02}$ or between outer cores as the field distribution of $\mathrm{LP}_{03}$, and a strong coupling also occurs between the two, four and six outer cores than the central core as the field distribution of $\mathrm{LP}_{11}$.

When the value of the central core is $3.79 \mu \mathrm{m}$ Figure 4 (c) there was a strong coupling between the central core to two of the six outer cores that have diameters close to the diameter of the central core and equal propagation constants as the field distribution of $\mathrm{LP}_{31}$, while the residual cores remain without any coupling and participate very little in the power exchange with the others. In addition to the the field distribution of $\mathrm{LP}_{01}$ and $\mathrm{LP}_{11}$.

Figure $5(\mathrm{a}-\mathrm{c})$ shows an evaluation of the power flow in each core of a system consists of the identical and nonidentical seven core. Fundamental modes with seven identical and non-identical cores and each core supports two propagation modes with initial input into the central core at the wavelength of $1.064 \mu \mathrm{m}$. For seven identical cores, the light blue line represents one of the central core mode, and the yellow line represents the other mode, while the rest of the colors represent the modes of the cores adjacent to the central core. Example Figure 5 (a) for seven identical, the black and yellow lines represent the modes of the central core, the black line represents one of the mode of the central core is decoupled with other outer cores $\left(\mathrm{LP}_{01}\right)$. While the yellow line represents other mode of the central core was a weak coupling with six outer cores represented by lines (blue, green, red, pink, yellow and black) as $\left(\mathrm{LP}_{02}\right)$ or a strong coupling between the six outer cores such as $\left(\mathrm{LP}_{11}\right)$ represented by lines expanded color (violet, light blue, blue, green, yellow and red lines) or strong coupling between two standing outer cores (black and yellow lines) or strong coupling between three standing outer cores (red, light blue and green) and strong coupling between four standing outer cores (green, pink, red and yellow). Example Figure 5 (b) for seven nonidentical, the coupling between three of the outer six cores have diameters close to that mode of the central core with black line, which is approximately similar to that shown as four-core PCF systems with identical cores that represented in lines (blue, blue, green and black) as $\left(\mathrm{LP}_{12}\right)$. The couplings between two outers cores represented in lines as (pink and yellow), the strong couplings between the other mode of the central core as yellow line with four outers cores represented in lines as (green, red, black and light blue), the couplings between the six outers cores represented that is extended in lines as (light blue, black, red, pink, green and yellow) as $\left(\mathrm{LP}_{11}\right)$. Example Figure 5 (c), The coupling was suppressed between one mode of the central core represents in yellow line and the outer core as $\mathrm{LP}_{01}$. The coupling between two of the outer six cores have diameters close to that of the central core, which is approximately similar to that shown as three-core PCF systems with identical cores that represented in lines as (black, red and green) or (yellow, red and green) as $\left(\mathrm{LP}_{31}\right)$. The couplings between two outer cores represented in lines as (red and green), the couplings between four outers cores represented in lines as (blue, black, pink and yellow), the couplings between the six outers cores are extended in lines as (pink, yellow, black, blue, light blue and red) as $\left(\mathrm{LP}_{11}\right)$.

Therefore, we conclude from the second and the third study that although there is little difference in the diameter of the cores, it introduces a considerable mismatch, which reduces the coupling between the cores. In some cases, a strong coupling occurred between the cores with different diameters because these cores have nearly identical propagation constants of different models, which could lead up to a zero phase mismatch between them. With these two studies, we were able to overcome the asymmetry in the structure and enhance the coupling between cores.

After extracting the refractive indices of the even and odd modes of each of the engineering structures show Table 1. and applying them in equation (4), it became possible to extract the coupling length for each structure with ease. In the case of cores are identical, we find the coupling length takes the value $1064 \mu \mathrm{m}$, but when there is a decrease in the central core diameter relative to the rest of the outer cores, we find a decrease in the value of the coupling length to $665 \mu \mathrm{m}$. An increase in the central core diameter relative to the rest of the outer cores found a decrease in coupling Length to $886.66 \mu \mathrm{m}$, as shown in Table 1 and Figure 6. On the other hand, we find that in the case of designing a system of asymmetric cores with an increase in its central core diameter without decreasing it from the rest of the neighboring hearts, it will have better results in terms of the coupling length and the coupling properties between the core diameter (or width). 


\section{Al-Nahrain Journal of Science}

ANJS, Vol.23 (3), September, 2020, pp. 49 - 60

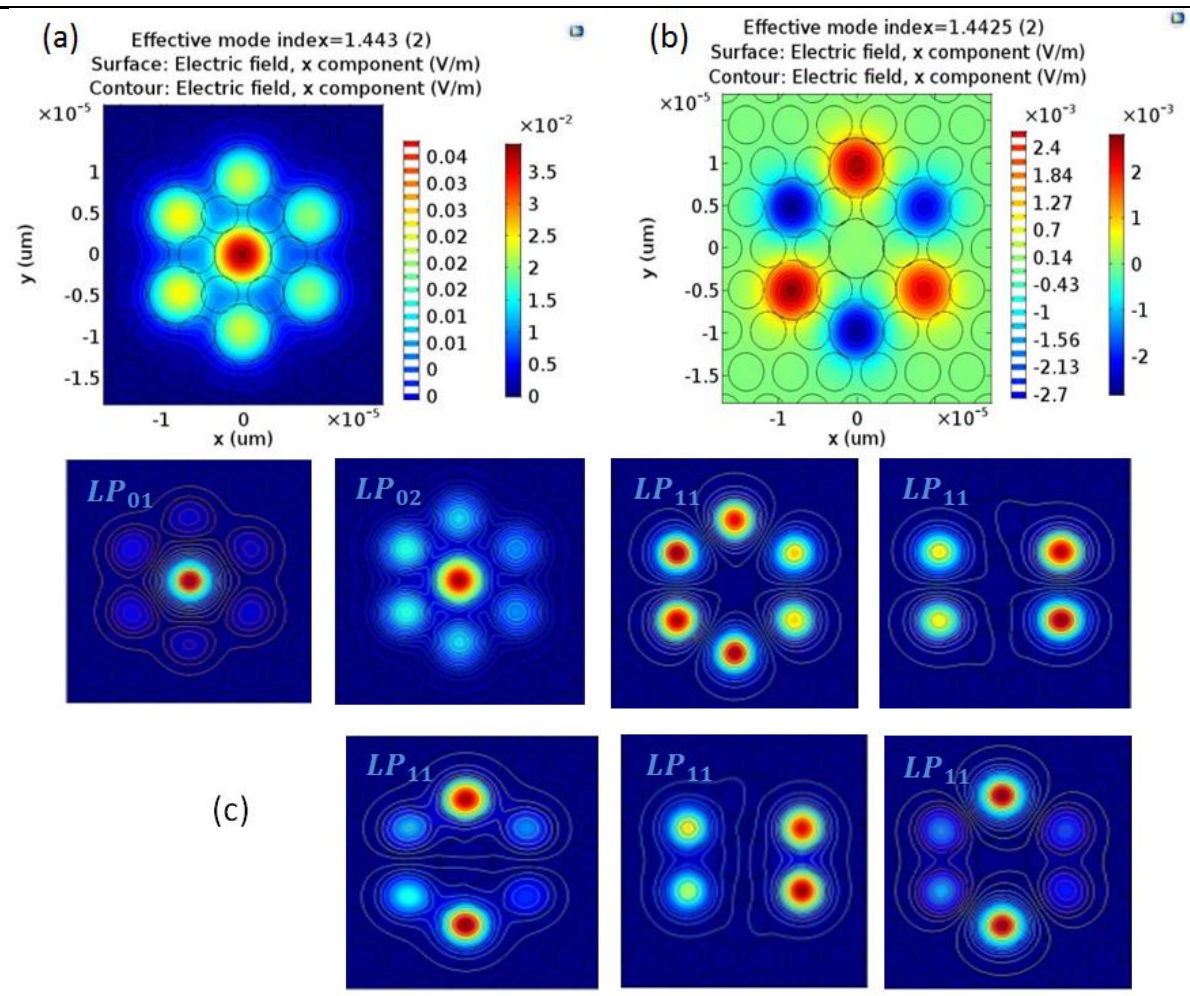

Figure 1. Cross-section of a coupled seven-core PCF with hexagonal lattice The structure parameters are $\Lambda=5.6 \mu \mathrm{m}$, $d=4.48 \mathrm{um}, d / \Lambda=0.8, \lambda=1.064 \mu \mathrm{m}$ when all seven cores have identical diameters $=3.5 \mu \mathrm{m}$. (a $\& \mathrm{~b})$ effective index and electric field along with the x-polarized component $\left(E_{x}\right),(\mathrm{c})$ the optical power in each core of a seven-core PCF.
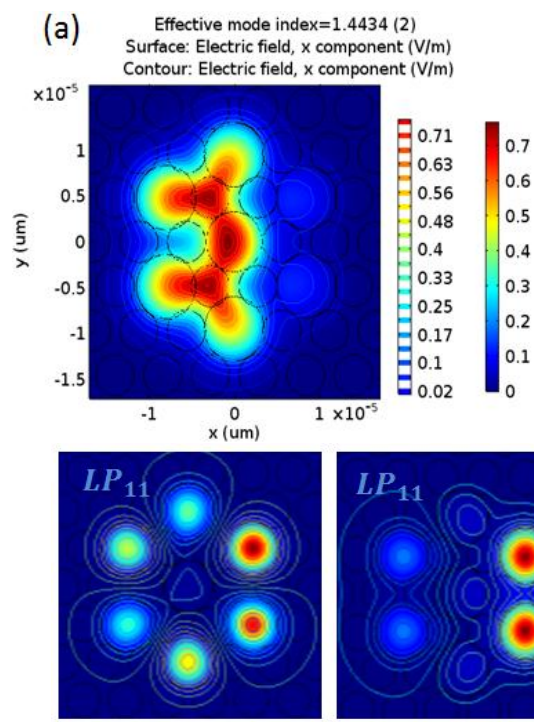

(c)
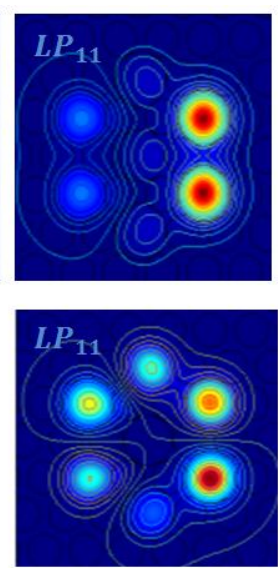

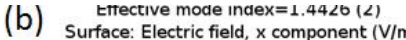
Surface: Electric field, $x$ component $(\mathrm{V} / \mathrm{m})$
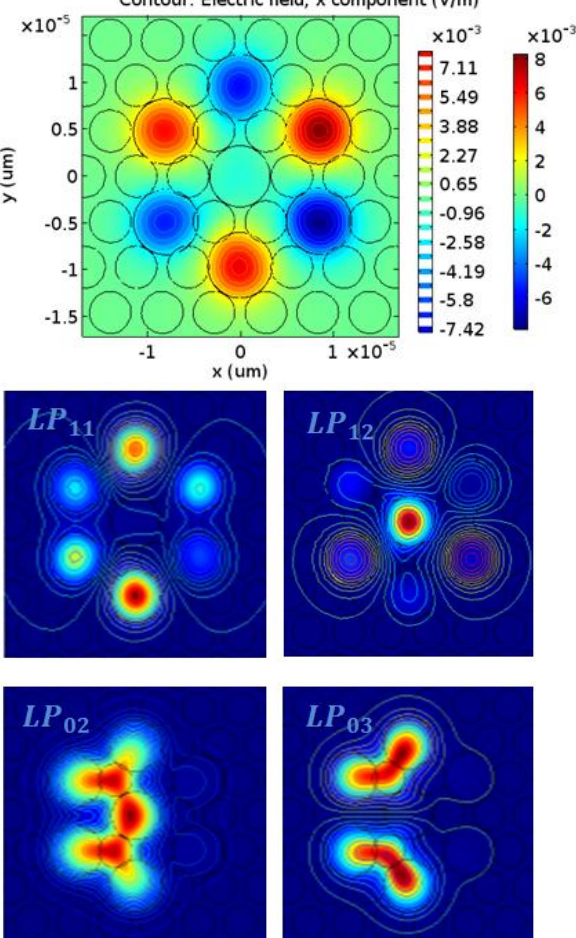

Figure 3. (a \& b) Effective index and electric field along with the $x$-polarized component $\left(E_{x}\right)$ for both even and odd modes of the non-identical seven-core PCF, for the central core have a diameter $3.27 \mu \mathrm{m}$ is slightly less than the diameters of the six outer cores of $3.5 \mu \mathrm{m}$. (c) The optical power in each core of a non-identical seven-core PCF when the input is initially to the central core. 


\section{Al-Nahrain Journal of Science}

ANJS, Vol.23 (3), September, 2020, pp. 49 - 60
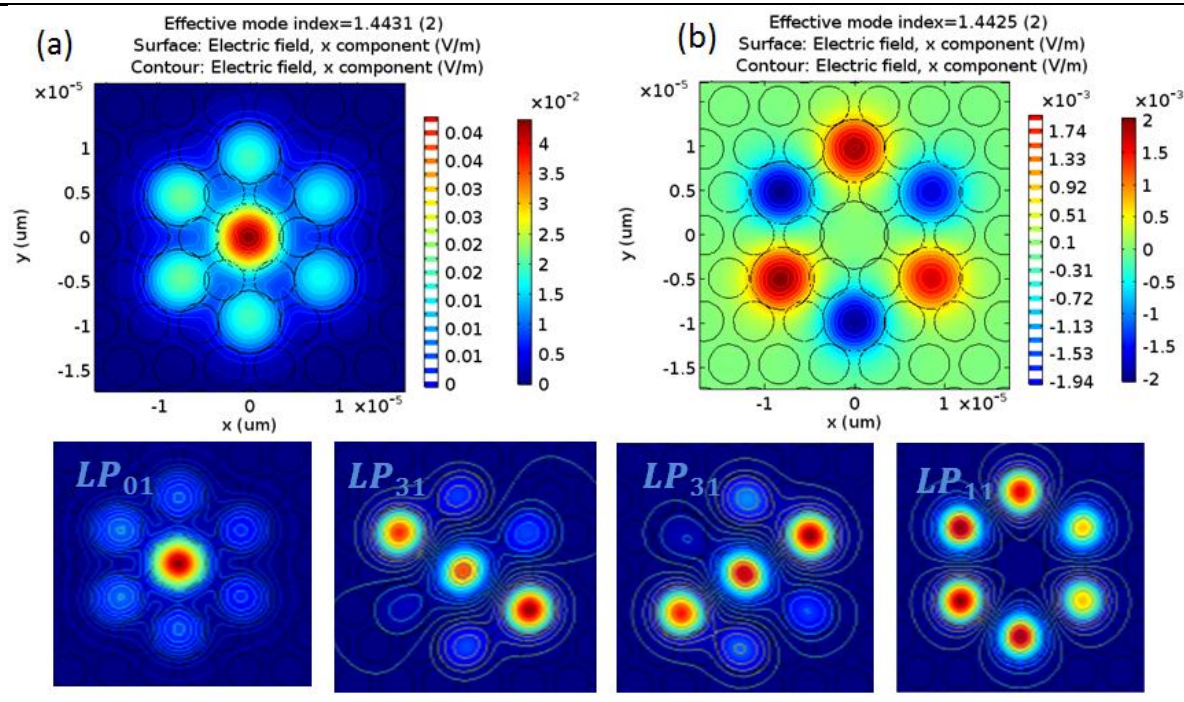

(c)
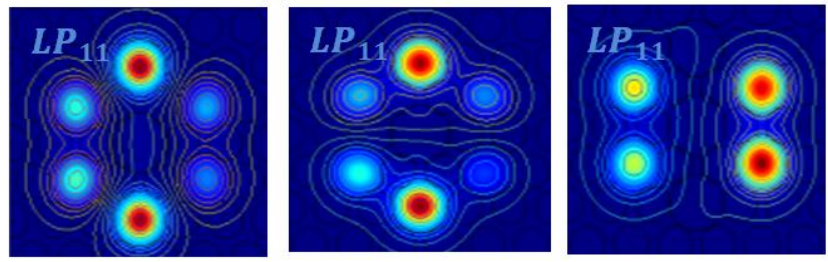

Figure 4. (a \& b) Effective index and electric field along with the $x$-polarized component $\left(E_{x}\right)$ for both even and odd modes of the non-identical seven-core PCF, for the central core have a diameter $3.79 \mu \mathrm{m}$ is slightly larger than the diameters of the six outer cores of $3.5 \mu \mathrm{m}$. (c) The optical power in each core of a non-identical seven-core PCF when the input is initially to the central core.
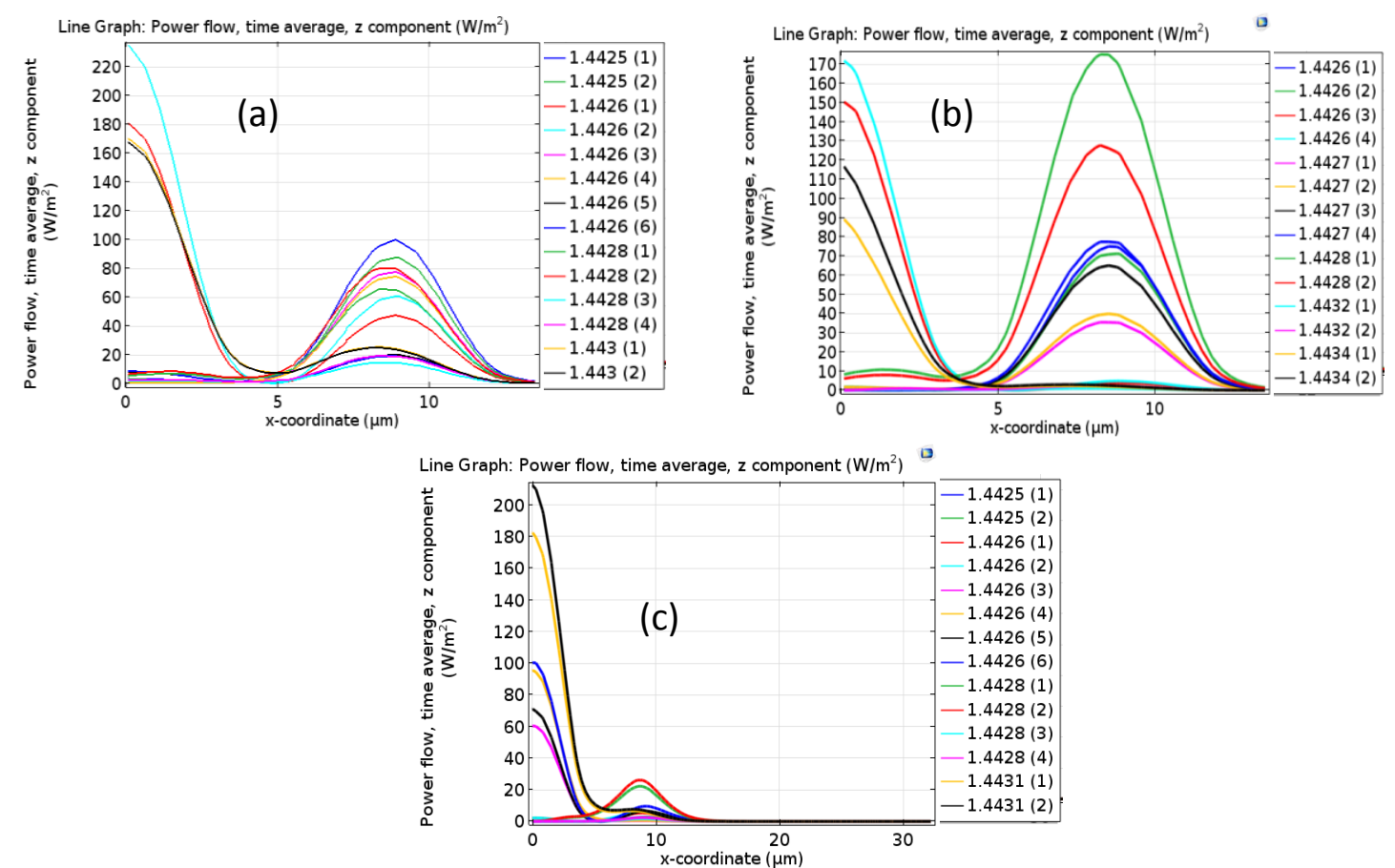

Figure 5. Evaluation of the optical power in each core for identical displays in (a) and non-identical seven cores PCF coupler display in (b and c) when the input is initially to the central core. 


\title{
Al-Nahrain Journal of Science
}

\author{
ANJS, Vol.23 (3), September, 2020, pp. 49 - 60
}

Table 1. Represents the numerical simulation results for three different configurations in terms of design, one of which is identical core of $3.5 \mu \mathrm{m}$ and two non-identical cores of $3.27 \mu \mathrm{m}$ and $3.79 \mu \mathrm{m}$ at wavelength of $1064 \mathrm{~nm}$.

\begin{tabular}{|c|c|c|c|c|c|}
\hline Core diameter & Even mode & Odd mode & $\begin{array}{c}\text { Difference in the effective } \\
\text { index for even and odd modes }\end{array}$ & $\begin{array}{c}\text { Coupling } \\
\text { length }(\boldsymbol{\mu m})\end{array}$ & $\begin{array}{c}\text { Coupling } \\
\text { coefficient }(\boldsymbol{\mu m})^{-\mathbf{1}}\end{array}$ \\
\hline $3.27 \mu \mathrm{m}$ & 1.4434 & 1.4426 & 0.0008 & 665 & 0.0023 \\
\hline $3.5 \mu \mathrm{m}$ & 1.443 & 1.4425 & 0.0005 & 1064 & 0.00147 \\
\hline $3.79 \mu \mathrm{m}$ & 1.4431 & 1.4425 & 0.0006 & 886.66 & 0.0017 \\
\hline
\end{tabular}

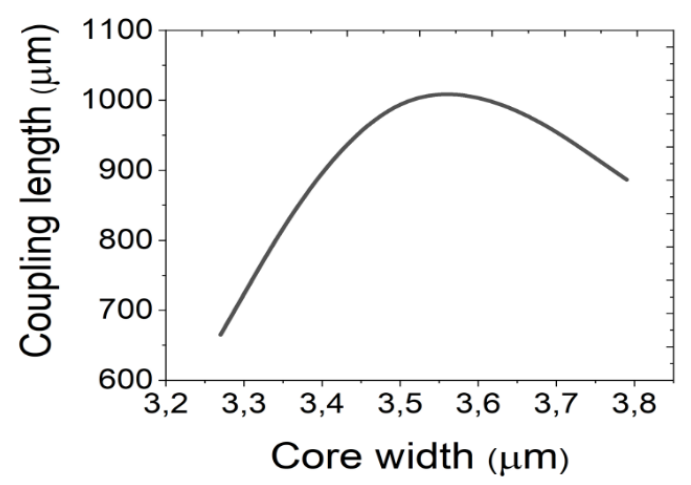

Figure 6. The relation between the core width and the coupling length, shows the coupling lengths decresed as the core width increase.

\section{A Effect the Core Separation}

We try to increasing the cores separation of the seven-core PCF has a strong effect on the coupling strength between the cores and the coupling length. A slight change in core diameters as well as the core separation can cause change in modal field. In case the seven-core are identical, irregularity in the core separation will affect strong coupling between cores and then the coupling length as shown in Figure 7 (a \& b), that represent the results for identical cores for different core separation, such as $(2.5$ $\mu \mathrm{m}$ and $1.5 \mu \mathrm{m})$. We found from the numerical results; for identical cores when core diameter remain constant, any increase in the cores spacing as $2.5 \mu \mathrm{m}$ (a and c) causes an increase in the mode fields of cores consequently, the coupling between cores are nearly weak. By decreasing the core separation as $1.5 \mu \mathrm{m}$ ( $\mathrm{b}$ and d) causes increase in the coupling between the central core and two outer cores or between the outers core only.

For seven identical cores $(3.5 \mu \mathrm{m})$ and the cores spacing as $2.5 \mu \mathrm{m}$ as in Figure 7 (a \& c), shows the black line represents one of the central core mode, and the yellow line represents the other mode. The coupling between one mode of the central core (yellow line) was suppressed and the outer cores as LP01. We found a weak coupling between one mode (yellow line) of the central core (black line) and six outer cores represented by lines as (blue, green, red, pink, yellow and green) such as $\left(\mathrm{LP}_{02}\right)$ or a strong coupling between two outer cores such as $\left(\mathrm{LP}_{11}\right)$ represented by lines as (yellow and black) or a strong coupling between three outer cores represented by lines as (light blue, red, green) as $\left(\mathrm{LP}_{11}\right)$ or a strong coupling between the four outer cores such as $\left(\mathrm{LP}_{11}\right)$ represented by lines expanded color (violet, light blue, black, blue, and red lines) as $\left(\mathrm{LP}_{11}\right)$. For the cores spacing $1.5 \mu \mathrm{m}$ as in Figure 7 (b \& d), we found there was no coupling between the black line that represents one of the central core mode and six outer as LP01. Also we found a weak coupling between the yellow line which represents one mode of the central core and two of outer cores, which is approximately similar to that shown as three-core PCF systems with identical cores that represented in lines as (blue and black) as $\left(\mathrm{LP}_{31}\right)$. Or we found a strong coupling between two outer cores represented by lines as (pink and yellow) as $\left(\mathrm{LP}_{11}\right)$ or we found a strong coupling between three outer cores represented by lines as (red, green and blue) as $\left(\mathrm{LP}_{11}\right)$ or a coupling between four outer cores represented by lines as (blue light, black, red and pink) as $\left(\mathrm{LP}_{11}\right)$ or a weak coupling between six outer cores represented by lines expanded color (light blue, red, pink, green, yellow and black lines) as $\left(\mathrm{LP}_{11}\right)$. For the cores spacing $1.5 \mu \mathrm{m}$, Figure 8 (down).

For non-identical cores of $(3.27 \mu \mathrm{m})$ and $(3.79 \mu \mathrm{m})$, the change in the cores separation strongly depended on the slightly change of the central core decrease or increase to the mode fields of cores closer or farther away.

A slight reduction in the core diameter as $(3.27 \mu \mathrm{m})$ can reduce the number of modes and also leads to increasing the spacing between the mode fields of outer cores causing to weak mode coupling between cores shown in Figure 8 ( $\mathrm{a} \& \mathrm{~b}$ ). For the cores spacing as $2.5 \mu \mathrm{m}$ as in Figure 8 (a \& c), we found the reduce in the core diameter, may leads to strong coupling between one the modes of the central core (black line) and four outer cores as in Figure 8 (a \& c), which is approximately similar to that shown as five-core PCF systems with identical cores that represented in lines (blue, light blue, yellow and pink) 


\section{Al-Nahrain Journal of Science}

ANJS, Vol.23 (3), September, 2020, pp. 49 - 60

as $\left(\mathrm{LP}_{31}\right)$. We found also the coupling between one mode of the central core (yellow line) was suppressed and the outer cores as $\mathrm{LP}_{01}$. Or we found a strong coupling between two outer cores represented by lines as (black and yellow) as $\left(\mathrm{LP}_{11}\right)$ or we found a strong coupling between three outer cores represented by lines as (blue, green and red) as $\left(\mathrm{LP}_{11}\right)$ or a coupling between four outer cores represented by lines expanded color (pink, light blue, blue and green) as $\left(\mathrm{LP}_{11}\right)$. For the cores spacing $1.5 \mu \mathrm{m}$ as in Figure 8 (b \& d), we found strong coupling between one of the central core mode (black line) and three outer cores when their diameters are almost all alike. This is similar to a system consisting of four-core with identical cores as $\left(\mathrm{LP}_{12}\right)$ and represented by lines as (blue, pink and yellow). We also found weak coupling between one of the central core mode (yellow line) and three outer cores when their diameters are almost all similar. This is similar to a system consisting of four-core with identical cores as $\left(\mathrm{LP}_{12}\right)$ and represented by lines as (light blue, black and pink). Or we found a strong coupling between two outer cores represented by lines as (black and yellow) as $\left(\mathrm{LP}_{11}\right)$ or we found a strong coupling between three outer cores represented by lines as (red, blue and green) as $\left(\mathrm{LP}_{11}\right)$ or a coupling between four outer cores represented by lines expanded color (light blue, yellow, red and green) as $\left(\mathrm{LP}_{11}\right)$.

A slight increase in core diameter as $(3.79 \mu \mathrm{m})$ will increase the number of modes and reduce the spacing between the mode fields of outer cores causing to strong mode coupling between cores as in Figure 9 (a \& b). For the cores spacing as $2.5 \mu \mathrm{m}$ as in Figure 9 (a \& c), we found the coupling was suppression between one the modes of the central core (black line) and six outer cores.
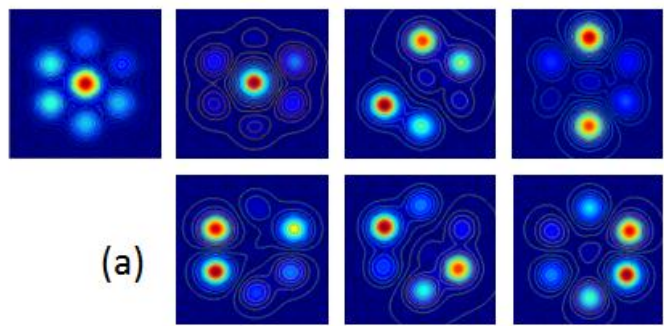

(a)
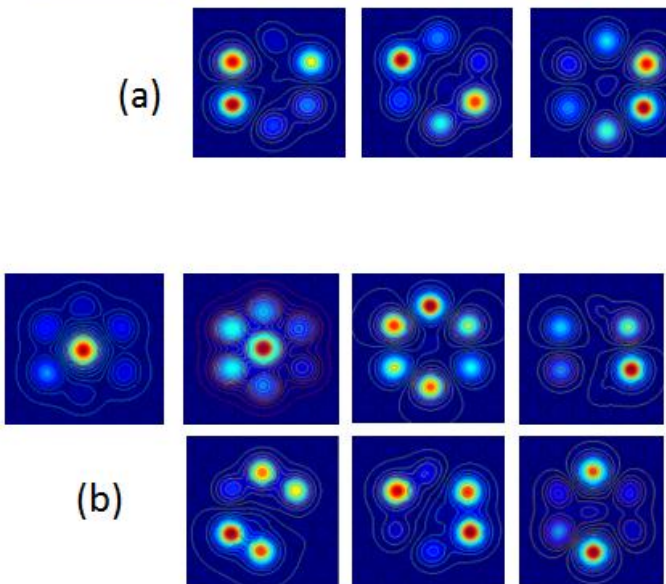

We also found strong coupling between one of the central core mode (yellow line) and two outer cores when their diameters are almost. This is similar to a system consisting of three-core with identical cores as $\left(\mathrm{LP}_{31}\right)$ and represented by lines as (red and green). The coupling between two outer cores represented in lines as (black and yellow), or the coupling between three outers cores represented in lines as (blue, red and green), or the coupling between four outers cores represented in lines as (blue, black, pink and yellow), the coupling between five outers cores represented in lines as (light blue, blue, pink, black and yellow) as $\left(\mathrm{LP}_{11}\right)$. For the cores spacing as $1.5 \mu \mathrm{m}$ as in Figure 9 (b \& d), we found the coupling was suppressed between the modes of the central core and the outer cores. We found also the strong coupling between two outer cores represented in lines as (black and yellow), or the coupling between three outers cores represented in lines as (red, yellow and green), or the coupling between four outers cores represented in lines as (light blue, pink, pink and blue), the strong coupling between six outers cores represented in lines as (red, green, black, yellow, light blue, blue) as $\left(\mathrm{LP}_{11}\right)$.

Table 2 and Figure 10 describes a scheme to illustrate the relationship between coupling length and core separation for different structures, for example, identical structure $3.5 \mu \mathrm{m}$ displays in the black line and nonidentical structures $3.27 \mu \mathrm{m}$ display in the blue line and $3.79 \mu \mathrm{m}$ display in the red line. Therefore, a slight change in core diameter and changing the cores separation can sufficiently limit the coupling between the guided mode of cores to achieve a strong transmission.
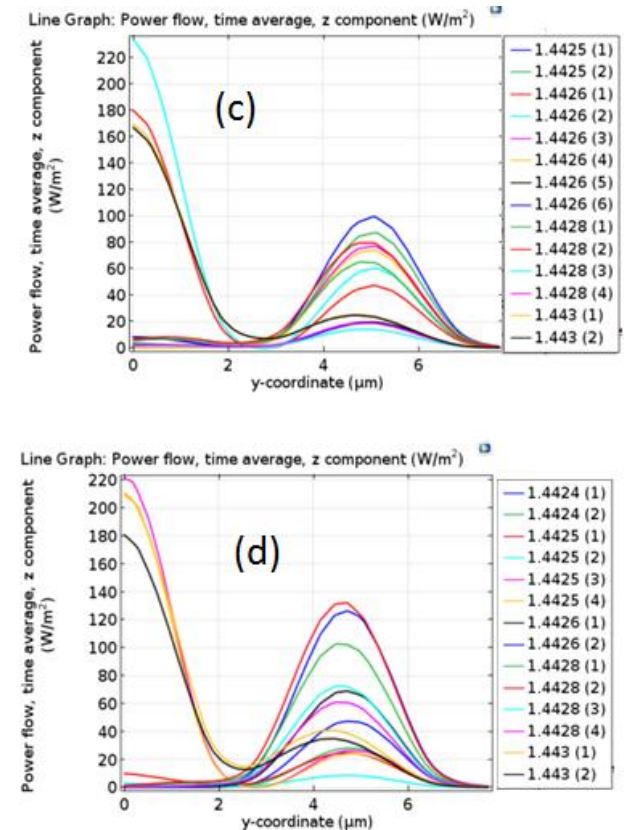

Figure 7. Contour: Electric field, along $x$-component of seven supermodes among the fourteen supermodes of PCF crosssection, display in $(\mathrm{a} \& \mathrm{~b})$ for case identical cores $(3.5 \mu \mathrm{m})$ from up $(2.5 \mu \mathrm{m})$ to down $(1.5 \mu \mathrm{m})$. (c \& d) represent the evaluation of $(\mathrm{a} \& \mathrm{~b})$ when the input is initially to the central core. 


\section{Al-Nahrain Journal of Science}

ANJS, Vol.23 (3), September, 2020, pp. 49 - 60
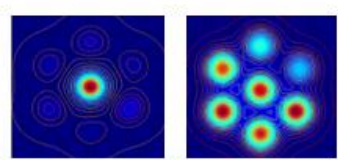

(a)
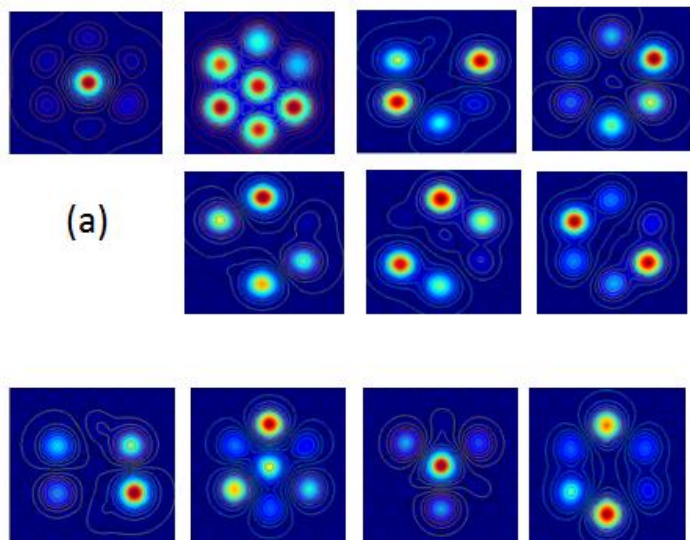

(b)
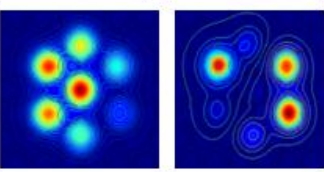
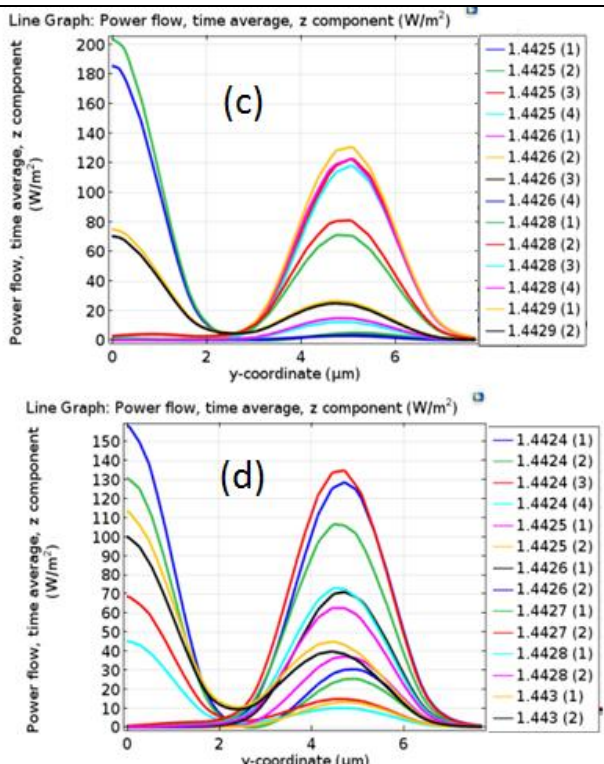

Figure 8. Contour: Electric field, along $x$-component of seven supermodes among the fourteen supermodes of PCF crosssection, display in $(\mathrm{a}$ and $\mathrm{b})$ for case non-identical cores $(3.27 \mu \mathrm{m})$ for different core separation from up $(2.5 \mu \mathrm{m})$ to down $(1.5 \mu \mathrm{m})$. (c \& d) represent the evaluation of ( $\&$ b) when the input is initially to the central core.

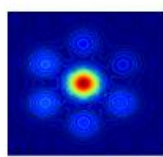

(a)
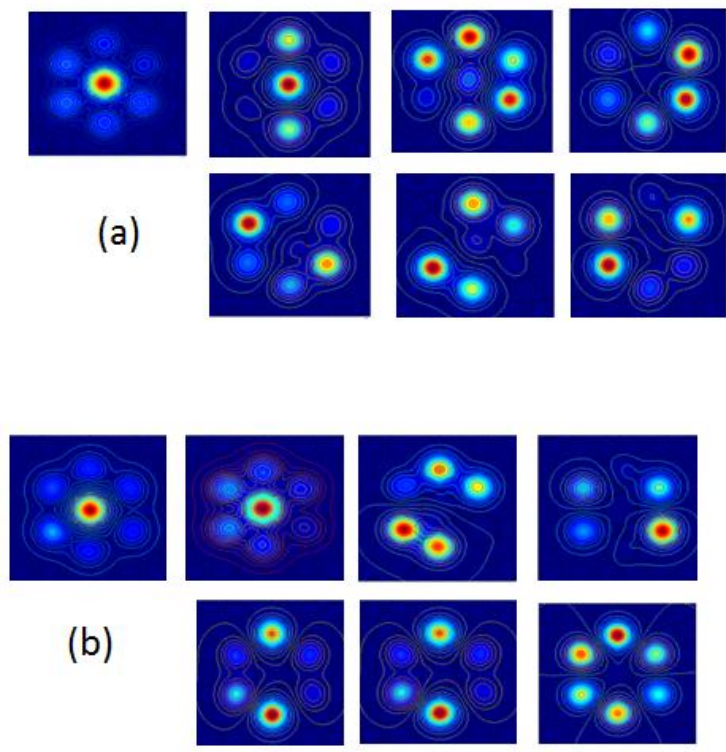
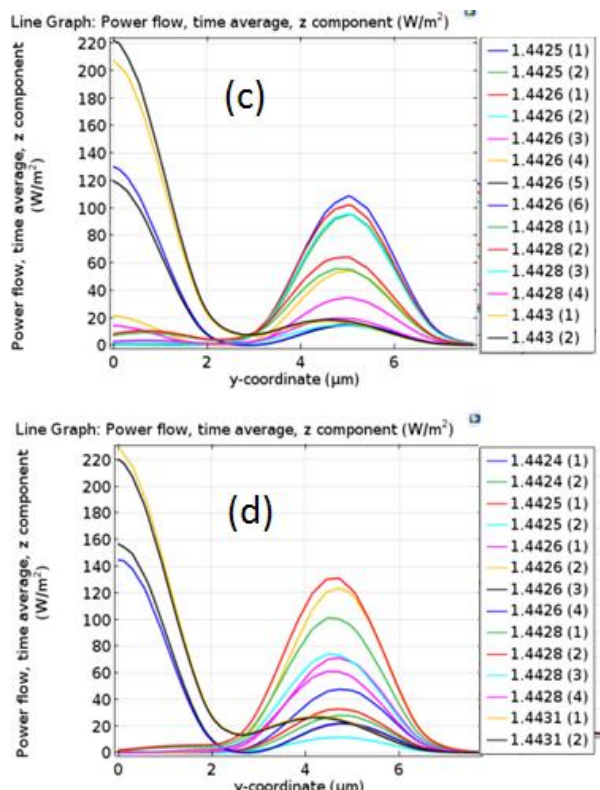

Figure 9. Contour: Electric field, along $x$-component of seven supermodes among the fourteen supermodes of PCF crosssection, display in (a \& b) for case identical cores $(3.79 \mu \mathrm{m})$ for different core separation from up $(2.5 \mu \mathrm{m})$ to down $(1.5 \mu \mathrm{m})$. $(c \& d)$ represent the evaluation of ( $\&$ b) when the input is initially to the central core.

Table 2. represents the numerical simulation results for three different configurations in terms of design and core separation, one of which is identical core of $3.5 \mu \mathrm{m}$ and two non-identical cores of $3.27 \mu \mathrm{m}$ and $3.79 \mu \mathrm{m}$ and the core separation was varied from $(1.5 \mu \mathrm{m})$ to $(2.5 \mu \mathrm{m})$ at wavelength of $1064 \mathrm{~nm}$.

\begin{tabular}{|c|c|c|}
\hline Core diameter & $\begin{array}{c}\text { Difference in the effective refractive index for } \\
\text { even and odd modes }\end{array}$ & Coupling length $(\boldsymbol{\mu m})$ \\
\hline $3.27 \mu \mathrm{m}$ & $\begin{array}{c}0.0006,0.0008, \text { and } 0.0004 \text { at core separation } \\
1.5(\mu \mathrm{m}), 2(\mu \mathrm{m}) \text { and } 2.5(\mu \mathrm{m})\end{array}$ & $886,66,665$, and 1330 \\
\hline $3.5 \mu \mathrm{m}$ & $\begin{array}{c}0.0006,0.0005 \text { and } 0.0004 \text { at core separation } \\
1.5(\mu \mathrm{m}), 2(\mu \mathrm{m}) \text { and } 2.5(\mu \mathrm{m})\end{array}$ & $886,66,1064$ and 1330 \\
\hline $3.79 \mu \mathrm{m}$ & $\begin{array}{c}0.0007,0.0006 \text { and } 0.0005 \text { at core separation } \\
1.5(\mu \mathrm{m}), 2(\mu \mathrm{m}) \text { and } 2.5(\mu \mathrm{m})\end{array}$ & $760,886.6$ and 1064 \\
\hline
\end{tabular}




\section{Al-Nahrain Journal of Science}

ANJS, Vol.23 (3), September, 2020, pp. 49 - 60

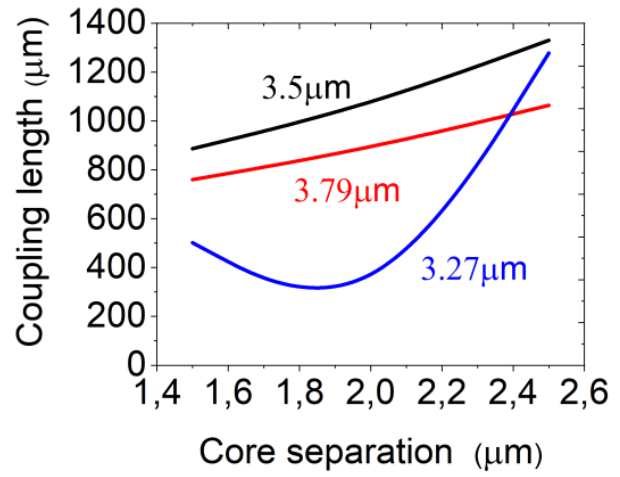

Figure 10. Changing in the coupling length with the core separation for different core diameter as $3.5 \mu \mathrm{m}$ display in the black line, $3.27 \mu \mathrm{m}$ display in the blue line and, $3.79 \mu \mathrm{m}$ display in the red line.

We attempted to clarify how non-uniformity in all seven core diameters $(3.2 \mu \mathrm{m}, 3.5 \mu \mathrm{m}, 3.47 \mu \mathrm{m}, 3.48 \mu \mathrm{m}$, $3.49 \mu \mathrm{m}, 3.51 \mu \mathrm{m}$ and $4.52 \mu \mathrm{m}$ of PCFs) affects the coupling properties between the cores as shown in Figure 11 ( $\mathrm{a} \& \mathrm{~b})$. We change the diameters of all seven cores a little bit more than others, to see intuitively how this affects the coupling properties between the seven cores. This is different from the first study, in which the diameter of all seven-core is identical, where the diameters of seven-core are $3.2 \mu \mathrm{m}, 3.5 \mu \mathrm{m}, 3.47 \mu \mathrm{m}, 3.48 \mu \mathrm{m}, 3.49$ $\mu \mathrm{m}, 3.51 \mu \mathrm{m}$ and $4.52 \mu \mathrm{m}$. We find from the numerical
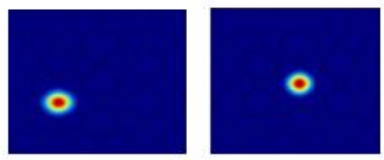

(a)
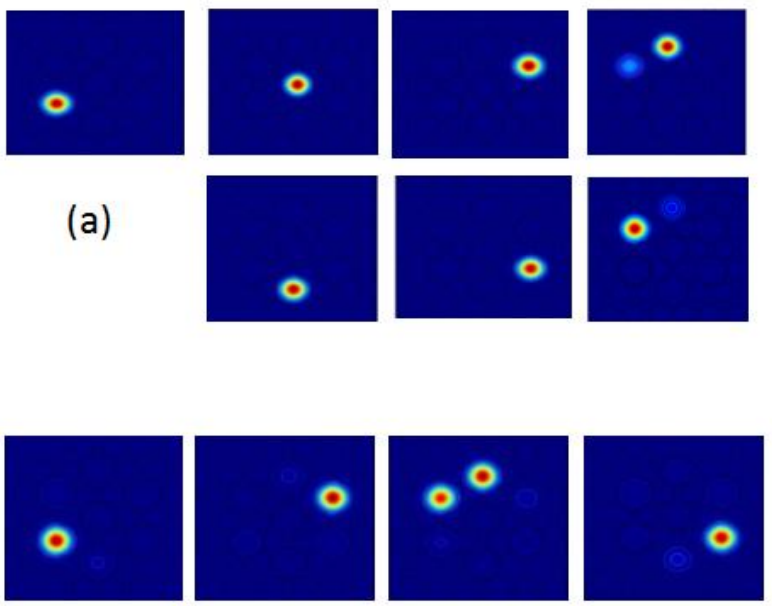

(b)
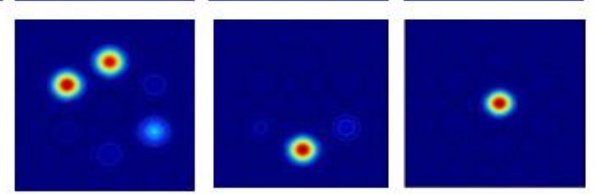

results that the anisotropy in all seven-core diameters radically reduces the coupling efficiency, and thus the modes of those cores become decoupled, remaining in their cores in isolation, as shown in Figure 11 (a), which shows the seven unique power distributions of a decoupled seven-core PCF with its fourteen non degenerate modes at $1.064 \mu \mathrm{m}$. Figure 11 (c), it represents an evaluation that there is no coupling between the modes of the central core and the modes of the outer cores and this very clearly in the figure, where the coupling efficiency was totally suppressed between seven cores. The reason is that the anisotropy in all diameters of the seven cores. This anisotropy creates a mode mismatch, and in turn impedes the coupling between these cores, such the cores become independent of their neighbors without interaction, and the light propagation in each core is also independent.

When increasing the wavelength to $1.55 \mu \mathrm{m}$, we found that the coupling efficiency improves slightly as in Figure 11 (b), and there is some coupling that occurs between the two cores. Otherwise, the cores remain independent of their adjacent. They tend to occupy only one site in a multicore PCF without/or with only a small negligible penetration into other adjacent cores. Figure 11 (d) represents an evaluation of the presence of some strong coupling between two outer cores although they are relatively different in their diameters. This result may be useful in applications, such as multiplexing and demultiplexing.
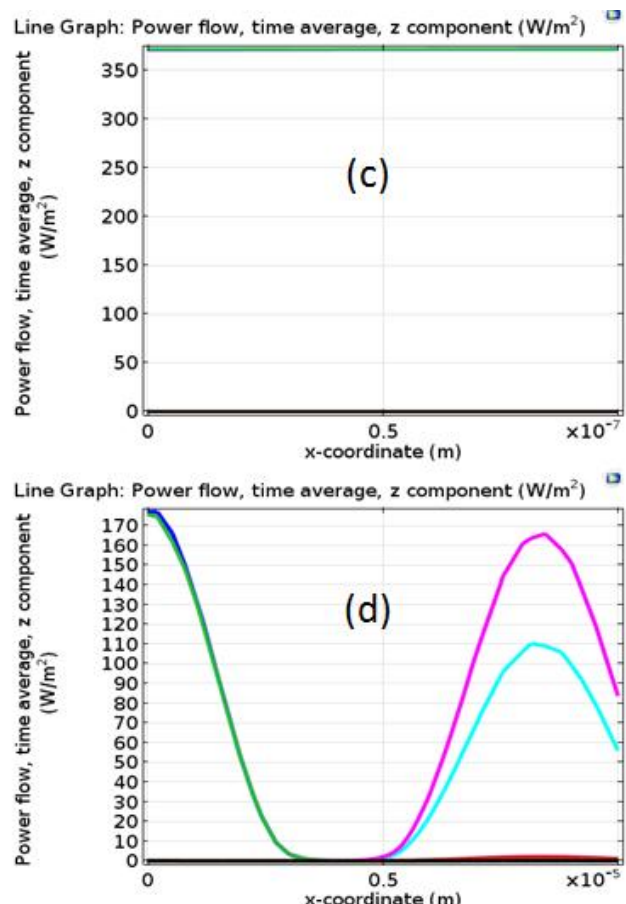

Figure 11. Distributions of the unique power for the seven-core with different wavelengths, $1.064 \mu \mathrm{m}$ (b) $1.55 \mu \mathrm{m}$, and (c \& d) represents an evaluation of the power as in ( $\mathrm{a} \& \mathrm{~b})$.

\section{Conclusions}




\title{
Al-Nahrain Journal of Science
}

\author{
ANJS, Vol.23 (3), September, 2020, pp. 49 - 60
}

In conclusion, we have numerically investigated the possibility of devising various forms of MCPCF by manipulating the geometrical design of the structure, such as a slight change in the central core diameter, and anisotropy in all core diameters of MCPCF. A slight change in the central core diameter to make the core nonidentical is sufficient to induce strong coupling between two or three seven-core PCF if compared to the identical cores where they are weakly coupled. The significant effect of anisotropy in all core diameters of MCPCF is a mode mismatch, which in turn, impedes the coupling between these cores, such that the cores become independent of their neighbors and the light propagation in each core independent. By increasing the wavelength, it is possible to overcome the problem of suppression of coupling between cores even if all cores different; these results may be useful in applications, such as coupler, splitter, multiplexing, and demultiplexing.

\section{References}

[1] Yu, X., Liu, M., Chung, Y., Yan M., Shum, P.: Coupling coefficient of two-core microstructured optical fiber. Opt. Commun. 260 (2006) 164-169.

[2] Rohini Priya, K., Raja, A. S., Sunda, D. S.: Modeling of twin core liquid filled photonic crystal fiber coupler with elliptical air holes. IJARTET 2394-3777(2015) 186-190.

[3] He, H., Wang, L.: Numerical analysis of birefringence and coupling length on dual-core photonic crystal fiber with complex air holes. Optik 124 (2013) 5941-5944.

[4] Khan, K. R., Wu, T. X.: Finite element modeling of dual-core photonic crystal fiber. ACES 3 (2008) 215.

[5] Saitoh, K., Sato, Y., Koshiba, M.: Coupling characteristics of dual-core photonic crystal fiber coupler. Opt. Express 24 (2003)3188-3195.

[6] Poli, F., Cucinotta, Selleri, A., S.: photonic crystal fibers: properties and applications. $1^{\text {st }}$ ed. Springer (2007).

[7] Yuan, C. L., Shi, J.: Supermode analysis of multicore photonic crystal fibers. Opt. Commun. 283 (2010) 2686.

[8] Reichenbach, K. L., Xu, C.: Independent core propagation in two-core photonic crystal fiber resulting from structural nonuniformities. Opt. Express $\mathbf{2 5}$ (2005) 10336-10348.

[9] Napierala, L. M., Ziolowicz, A., Pytel, A. N., Tenderenda, T., Nasilowski, T.: Cross talk analysis in multicore optical fibers by supermode theory. Opti. Lett. 41 (2016) 3759.

[10] Reichenbach, K. L., Xu, C.: Numerical analysis of light propagation in image fibers or coherent fiber bundles. Opt. Express. 15 (2007) 2151-2156.

[11] Zhou, J.: A non-orthogonal coupled mode theory for supermodes inside multicore fibers. Opt. Express 22 (2014) 10816-10824.
[12] Xu, Q., Luo, W., Li, K., Copner, N., Lin, S.: Design of polarization splitter via liquid and $\mathrm{Ti}$ infiltrated photonic crystal fiber. Crystals 9 (2019) 103.

[13] Andres, J., Dario, N., Gonzalez, Torres, E., P., Reyes, E.: Tunable Mode Converter Device Based on Photonic Crystal Fiber with a Thermo-Responsive Liquid Crystal Core. Photonics 7 (2020) 3.

[14] Wang, J., Zhang, Z., Li, S. Wang, S.: Microstructured optical fiber magnetic field sensor based on magnetic fluid filling. Modern Physics Letter B 33(2019) 1950380.

[15] Yu, Y., Sun, B.: Ultra-wide-bandwidth tunable magnetic fluid-filled hybrid connected dual-core photonic crystal fiber mode converter. Crystals 8 (2018) 95.

[16] Malka, D., Peled, A.,: Power splitting of $1 \times 16$ in multicore photonic crystal fiber. Appl. Surf. Sci. 417(2017) 34-39.

[17] Uthayakumar, T., Raja, R. V. J., Porsezian, K.: Alloptical steering of light through nonlinear twin-core photonic crystal fiber coupler at $850 \mathrm{~nm}$. J. Lightwave Technol. 13 (2012) 2110-2115.

[18] Uthayakumar, T., Raja, R. V. J., Porsezian, K., Grelu, P.: Impact of structural asymmetry on the efficiency of triple-core photonic crystal fiber for all-optical logic operation. J. Opt. Soc. Am. 32 (2015) 19201928.

[19] Wang, J., Nadkami, S. K.: The influence of optical fiber bundle parameters on the transmission of laser speckle patterns. Opti. Express 22 (2014) 8908-8918.

[20] Parto, M., Amen, M., M-ALI, M., Amezcua, R., LI, G., Christodoulides, D. N.: Systematic approach for designing zero-DGD coupled multicore optical fibers. Opt. Lett. 41 (2016) 1917-1920.

[21] C. Xia, M. A. Eftekhar, R. A. Correa, J. A.-Lopez, A. Schulzgen, D. Christodoulides and G. Li, Supermodes in coupled multicore waveguide structures, IEEE J. Quantu. Electron. 22 (2016) 4401212.

[22] Y. Amnon, Coupled-mode theory for guided-wave optics, IEEE Journal of Quantu. Electron. 9 (1973) 919.

[23] Mothe, N., Bin, P. D.: Numerical analysis of directional coupling in dual-core microstructured optical fiber. Opt. Express 18 (2009) 15778-15788.

[24] Ren, W, Tan, Z., Ren, G.: Analytical formulation of supermode in multicore fibers with hexagonally distributed cores. IEEE Photonic. Society 7 (2015) 7100311.

[25] Mohammed, D., and Mohammed, C.: Coupling mode of dual-core micro structure. J. Electrical and electronics engineering (2015).

[26] Mohammed, M.: Fem analysis of two-core photonic crystal fiber coupling characteristics. In: Computational Methods in Engineering Science, CEMS CONFERENCE 2019, ISBN: 978-83-7947386-1, pp.74-87, Kazimierz Dolny, Poland, November (2019). 


\section{Al-Nahrain Journal of Science}

ANJS, Vol.23 (3), September, 2020, pp. 49 - 60

www.biblioteka.pollub.pl/wydawnictwa.

[27] Miami, M., Ahmad, K. A.: Multicore Photonic Crystal Fibers Designing for Multiplexers and De-multiplexers Applications. Journal of Al-Rafidain Science, ISSN: 2664-2786, (2020).

\section{Acknowledgment}

This work was partially supported by the Republic of Iraq Ministry of High Education\& Scientific Research scholarship (MoHESR Grant No. 16408). And also author acknowledges the University of Muenster-department of physics, Germany to support. Comsol Multiphysics ver. 5.5 license number 6086097 . 